\title{
Developments in the Correlative Cryo Confocal Light Microscope (C3LM) at the Advanced Photon Source
}

\author{
Everett Vacek, Oliver Schmidt, Sunil Bean, Si Chen, Qiaoling Jin and Chris Jacobsen
}

Argonne National Lab, Lemont, Illinois, United States

In recent years correlative light and electron microscopy (CLEM) has emerged as a powerful tool in the investigation of biological samples [1,2]. By combining data from both visible light fluorescence and electron microscopy, CLEM provides a more complete picture of the inner workings of a biological sample. Furthermore, fluorescence microscopy can aid in screening samples greatly increasing the efficiency with which the electron microscope is used. To allow for examination in the vacuum of a transmission electron microscope, samples are often chemically fixed and dehydrated, but this can lead to both chemical and structural modification. Alternatively, cryogenic sample preparation preserves the sample closer to its naitive state and has thus become increasingly important in recent years [1].

X-ray microscopy provides capabilities that are complementary to electron microscopy, including the ability to image whole cells or tissue sections tens of micrometers thick, and improved sensitivity for trace element mapping [3]. The Bionanoprobe (BNP) at the Advanced Photon Source (a synchrotron light source providing bright $\mathrm{x}$-ray beams) combines cryogenic sample handling, X-ray fluorescence, tomography, and ptychography into a single instrument. It employs a sample transfer robot and cryogenic sample transfer system which prevents samples from ever leaving a cryogenic state once they are prepared [4]. It also allows rapid exchange of samples, greatly cutting down time spent not collecting data. As it stands one can simultaneously obtain x-ray ptychography images of cellular ultrastructure (sub-20 nm) and $\mathrm{x}$-ray fluorescence images of trace element distributions (sub-100 nm) [5]. Furthermore, the BNP also stands to benefit greatly from the upcoming APS-U upgrade which will increase X-ray beam brightness up to 1,000x. This increased signal will improve detection of trace elements as well as allow images to be acquired at faster rates.

The Correlative Cryo Confocal Light Microscope (C3LM) will add visible light fluorescence to the toolkit of capabilities at the BNP. The C3LM will use the same robotic transfer system as the BNP, keeping samples at cryogenic temperatures at all times and enabling easy interplay between the two systems. Samples will be mounted via robotic transfer onto motorized specimen stages in front of exchangeable objective lenses all of which will be housed in vacuum to prevent ice build-up. The rest of the microscope optics will be mounted on a viewport outside the vacuum chamber. All stages and motors inside the chamber will be operated by computer interface allowing for programmable data collection.

Confocal microscopy allows for thick samples to be imaged as well as for 3D fluorescence imaging via focus stacking. This feature of the C3LM will compliment both the tomographic and thick sample capabilities of the BNP, and provide a platform for 3D correlative microscopy. Similar to CLEM, the C3LM will not only expand the types of data attainable at the BNP but also it will help improve on the efficiency of the BNP's use. Having a separate light microscope which can handle the cryogenic samples used at the BNP will allow users to prescreen their samples in advance of scheduled beamtime.

Currently the microscope is being assembled. All stages and motors have been purchased and the vacuum system is nearing completion. After the vacuum system is finished, the next steps include assembling the external mount for the sample gripper and attaching the LN2 tank to the body of the vacuum chamber. Next, interior motors, stages and cooling equipment will be assembled and the sample gripper will be inserted. The last piece of equipment to be attached will be the exterior microscope optics to the external 
view port. The last step will constitute programming the motors and vacuum interlocks to provide a simple interface for users to operate the microscope.
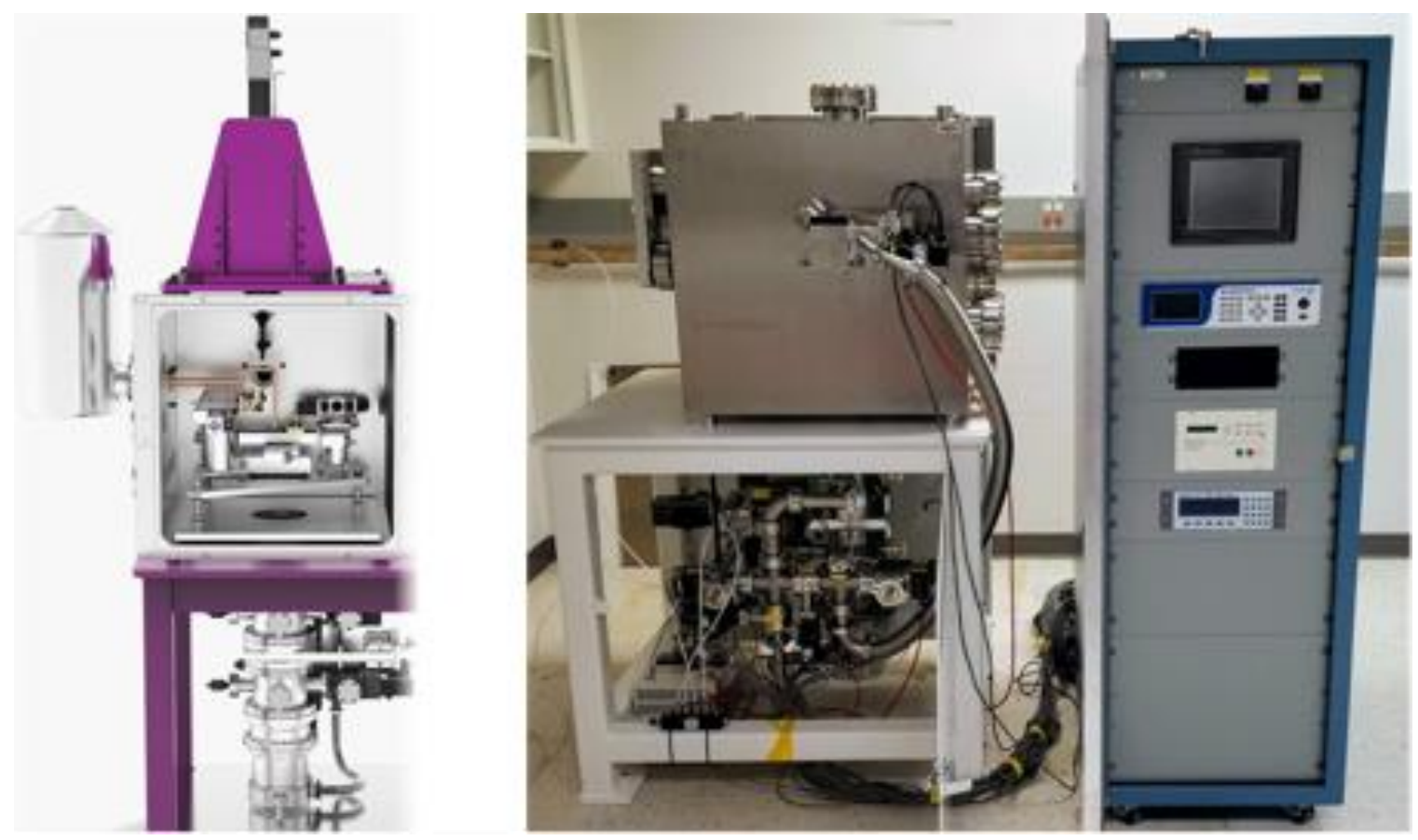

Figure 1. CAD mock-up of the completed C3LM alongside it in its current state of development (including the controllers for the motors, interlocks, and vacuum gauges).

\section{References}

[1] F. Moser et al, PNAS 11 (2019), p.4804

[2] J. Caplan et al., Current Opinion in Structural Biology 21, 686 (2011)

[3] C. Jacobsen, "X-ray Microscopy” (Cambridge University Press, 2020).

[4] S. Chen et al., J. Synchrotron Radiation 21 (2014), p. 66.

[5] J. Deng et al, Scientific Reports 7 (2017), p. 445. 